\title{
Biofilm formation by Streptococcus agalactiae: influence of environmental conditions and implicated virulence factors
}

\section{Roberto Rosini and Immaculada Margarit*}

Novartis Vaccines, Siena, Italy

\section{Edited by:}

Pietro Speziale, University degli

Studi di Pavia, Italy

Reviewed by:

Glen C. Ulett, Griffith University, Australia

Parish Paymon Sedghizadeh, University of Southern California, USA

\section{*Correspondence}

Immaculada Margarit, Novartis Vaccines, via Fiorentina 1, 53100

Siena, Italy

e-mail: immaculada.margarit_y_ ros@novartis.com
Streptococcus agalactiae (Group B Streptococcus, GBS) is an important human pathogen that colonizes the urogenital and/or the lower gastro-intestinal tract of up to $40 \%$ of healthy women of reproductive age and is a leading cause of sepsis and meningitis in the neonates. GBS can also infect the elderly and immuno-compromised adults, and is responsible for mastitis in bovines. Like other Gram-positive bacteria, GBS can form biofilm-like three-dimensional structures that could enhance its ability to colonize and persist in the host. Biofilm formation by GBS has been investigated in vitro and appears tightly controlled by environmental conditions. Several adhesins have been shown to play a role in the formation of GBS biofilm-like structures, among which are the protein components of pili protruding outside the bacterial surface. Remarkably, antibodies directed against pilus proteins can prevent the formation of biofilms. The implications of biofilm formation in the context of GBS asymptomatic colonization and dissemination to cause invasive disease remain to be investigated in detail.

Keywords: streptococcal infections, Streptococcus agalactiae, biofilms, group B streptococcus, pili/fimbriae/curli

\section{INTRODUCTION}

The beta-hemolytic Gram-positive Streptococcus agalactiae (Group B Streptococcus, GBS) is often encountered in the gastro-intestinal and the genital tract of healthy women as part of the normal flora. From this site, the bacteria can reach the newborn through the birth canal and cause sepsis and/or meningitis (Gibbs et al., 2004; Dando et al., 2014). GBS is also an important cause of morbidity and mortality in the elderly and in immuno-compromised adults. Primary manifestations of adult GBS disease include bacteremia, skin and soft tissue infections, pneumonia, osteomyelitis and urinary tract infections (Edwards et al., 2005; Skoff et al., 2009). GBS can also colonize the mammary gland of ruminants, where it is able to survive for long periods causing clinical and sub-clinical mastitis (Keefe, 1997).

GBS colonization and infection of target tissues requires the capacity of these bacteria to adhere and to persist in mucosal epithelial surfaces. In this habitat, the formation of biofilm-like communities could facilitate microbial survival and proliferation by enhancing resistance to host defenses and nutrient deprivation.

The present review summarizes recent studies investigating the capacity of GBS to form biofilm-like structures in vitro, how this mode of growth is affected by environmental conditions, and the contribution of adhesin virulence factors.

\section{EXPERIMENTAL EVIDENCE OF GBS BIOFILM FORMATION AND INFLUENCE OF ENVIRONMENTAL CONDITIONS}

Initial evidence suggesting that GBS could be implicated in the formation of biofilms came from studies by Donlan and Costerton where GBS bacteria were found on intrauterine devices in association with other known biofilm formers such as Staphylococcus aureus and Staphylococcus epidermidis (Donlan and Costerton, 2002).

Macroscopic assays were set up to investigate the biofilm forming capacity of GBS strains belonging to different lineages (Rinaudo et al., 2010). According to these type of assay, bacteria are cultured under static conditions in the wells of plastic tissue culture plates and, after several washes, microbial threedimensional structures are stained with crystal violet or similar compounds (O’Toole et al., 2000).

More restrictive experimental methods have recently been set up to better discriminate between GBS weak and strong biofilm formers. One of these approaches mimics fluid circulation in the host by using flow conditions in laminar chamber systems (Konto-Ghiorghi et al., 2009). An alternative multiwellbased protocol is based on plate incubation under shaking and removal of non-attached bacteria by extensive washing, followed by replacement of the growth medium (D'Urzo et al., 2014).

Environmental conditions are known to strongly influence the capacity to form biofilm by many bacterial species (Froeliger and Fives-Taylor, 2001; Moscoso et al., 2006; Manetti et al., 2007). Several studies have investigated GBS in vitro biofilm formation using one of the above described methods and different growth media, with contrasting results. For instance, Kaur et al. (2009), Borges et al. (2012), and Yang et al. (2012) investigated biofilm production under neutral and acidic $\mathrm{pH}$ conditions. They found larger biofilm amounts at $\mathrm{pH} 6.5$ compared to $\mathrm{pH}$ 4.2, probably due to poor bacterial growth at low $\mathrm{pH}$. By contrast, Ho et al. (2013) found that low $\mathrm{pH}$ induced biofilm formation in nutrient-limited chemically defined medium (M9YE) and not in rich media like Todd-Hewitt Broth (THB). Konto-Ghiorghi et al. (2009) reported that a uniform biofilm was produced only on 
Luria Broth and RPMI 1640 supplemented with 1\% glucose. The need for glucose for GBS biofilm formation was confirmed by Rinaudo et al. who also demonstrated that this sugar does not affect planktonic bacterial growth (Rinaudo et al., 2010). Previous studies had shown that in the GBS related species Streptococcus pyogenes the glucose biofilm enhancing effect was the direct result of acidification due to metabolic production of organic acids (Manetti et al., 2010). Evidence that acidic pH and not glucose concentration was the environmental signal driving GBS biofilm formation in vitro was obtained by D'Urzo et al. (2014). The authors tested a wide panel of strains in both buffered or non-buffered nutrient-rich (THB) and nutrient-limited (RPMI) media, in the presence or absence of glucose. Strong biofilm formation was observed only in glucose-containing non-buffered media and in low $\mathrm{pH}$ media even in the absence of glucose. In an in vivo setting, exposure of GBS to the acidic milieu of the vagina could be the signal sensed by the bacteria to grow in a sessile mode in this site. In this context, temporal shifts in GBS loads were recently observed in a mouse model of vaginal colonization (Carey et al., 2014), and have also been reported in humans (Hansen et al., 2004). It is tempting to speculate that $\mathrm{pH}$ variations and consequently GBS biofilm formation on epithelial cells could affect GBS carriage fluctuations.

Therefore, the discrepancies between the different studies in the observed capacity of GBS strains to form biofilm-like structures in vitro can possibly be explained by the use of different types of assays and growth conditions.

\section{CONTRIBUTION OF PILI AND OTHER SURFACE VIRULENCE FACTORS TO GBS BIOFILM FORMATION}

Long filamentous structures protruding from the surface of Gram-positive bacteria were discovered in the last decade (TonThat and Schneewind, 2004; Soriani and Telford, 2010). These structures resemble the pili found in Gram-negative bacteria, although in Gram-positives pilus protein components are linked by covalent bonds.

Pili in S. agalactiae were discovered during screening of multiple genomes for surface-exposed protein antigens as possible vaccine targets (Lauer et al., 2005). By SDS-PAGE, the pilus polymers appeared as a ladder of bands ranging from $150 \mathrm{kDa}$ to beyond the resolution of the gels, while immune electron microscopy revealed long appendages protruding outside the capsule that covers the bacterial surface (Figure 1). The genes encoding the GBS pilus machinery are clustered in three related genomic islands (Islands PI-1, $-2 \mathrm{a}$, and -2b) located in two separate loci flanked by direct repeats and conserved genes. All islands contain three genes encoding the pilus components, i.e., one backbone protein essential for pilus assembly (BP) and two accessory proteins (AP1 and AP2), plus two genes encoding sortase enzymes catalyzing the covalent linkage of the pilus proteins into long polymers (Dramsi et al., 2006; Rosini et al., 2006). The pilus proteins of PI- 1 and PI-2b differ by very few amino acids, while $\mathrm{PI}-2 \mathrm{a}$ is more variable with seven alleles described both for the BP and the AP1 presenting sequence identities between $48-98 \%$ for $\mathrm{BP}$ and $87-98 \%$ for AP2. Remarkably, mouse immunization with BP-1, BP-2b, and AP1-2a conferred protection against infection with a large panel of virulent strains, and at least one of the three

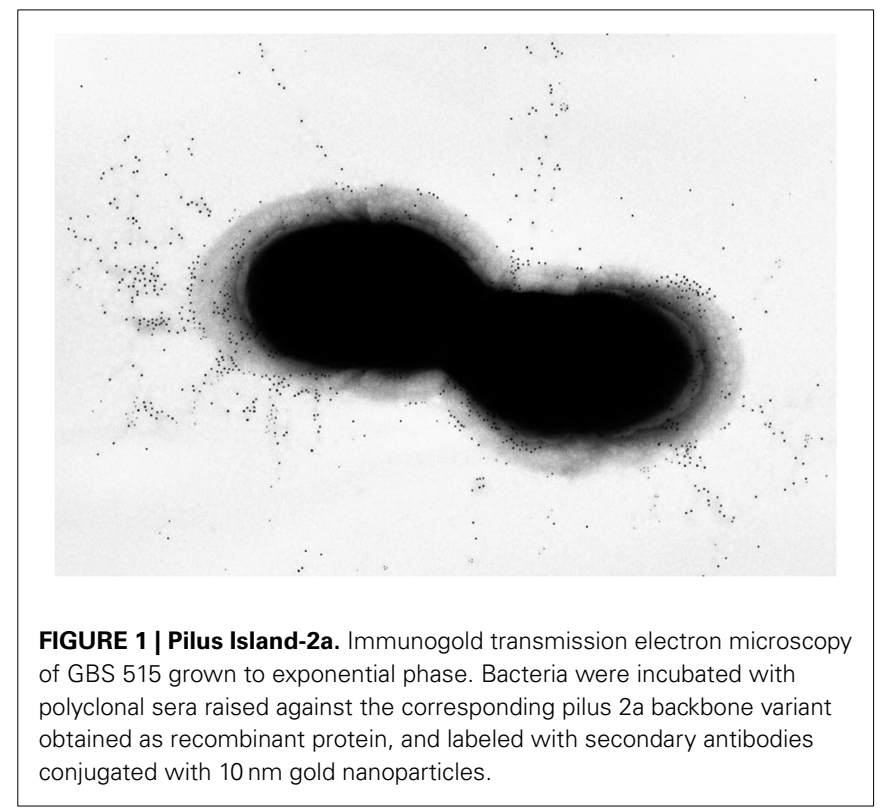

islands is present in all GBS (Margarit et al., 2009; Madzivhandila et al., 2011; Martins et al., 2013).

The discovery of pili in Gram-positive pathogens raised the question on the possible role of these highly surface-exposed structures in host colonization and infection. Pioneering evidence for their involvement in cell adhesion and biofilm formation was obtained in S. pyogenes (Manetti et al., 2007).

Studies using GBS isogenic mutants lacking pilus 2a components or the sortase enzymes responsible for pilus polymerization and cell wall attachment, indicated a role of pili in host cell contact and in the formation of multi colony three-dimensional structures on abiotic surfaces (Konto-Ghiorghi et al., 2009; Rinaudo et al., 2010). These biofilm-like structures were also analyzed by confocal laser scanning microscopy where the wild-type GBS515 strain bearing pilus variant $2 \mathrm{a}$ and an isogenic mutant unable to assemble pili, were seeded on glass polylysine-coated coverslips and stained with a fluorescent dye. As shown in Figure 2, the wild-type strain formed structured multilayered aggregates of surface-adherent bacteria resembling a mature biofilm, while the deletion mutant did not.

Remarkably, antibodies directed against the backbone of pilus $2 \mathrm{a}$ and its main ancillary protein inhibited the formation of these biofilm-like structures in a dose dependent manner, while antibodies against the small ancillary protein located at the pilus base near the cell wall, did not show any effect (Rinaudo et al., 2010). The same study investigated biofilm formation by 289 GBS clinical isolates using the above described crystal violet assay under static conditions. A correlation between the high surface exposure of pilus $2 \mathrm{a}$ and the biofilm formation phenotype was observed.

In the more recent study by D'Urzo et al., the formation of biofilm-like structures in vitro by 389 GBS isolates was investigated under more stringent conditions to better discriminate between weak and strong biofilm formers (see above). Also in this case, a high variability among strains was observed both in pilus expression and in the capacity to form biofilms, even when 


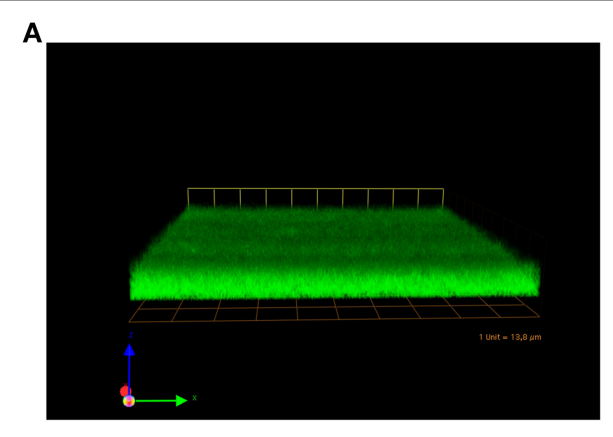

FIGURE 2 | Pilus Island-2a involvement in the formation of biofilms in vitro. Confocal scanning laser microscopy micrographs of biofilm development by GBS 515 (A) and its mutant derivative containing an in-frame

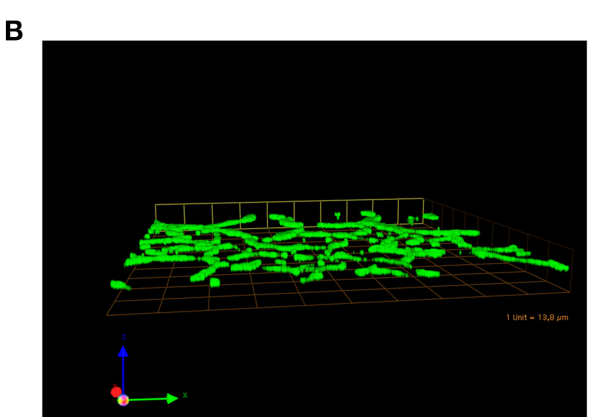

deletion in the pilus backbone gene (Rosini et al., 2006) (B). Bacteria were grown on glass coverslips under static conditions at $37^{\circ} \mathrm{C}$ for $72 \mathrm{~h}$, fixed and stained with SYTO-9 prior to confocal analysis (magnification 60x). they belonged to the same serotype or MLST phylogenetic lineage. A subset of serotype III strains belonging to the hyper virulent lineage ST-17 harboring both pilus 1 and 2b was shown to form stronger biofilms than all other tested strains, particularly at low pH (D'Urzo et al., 2014). Importantly, ST-17 strains are the most frequent cause of late-onset neonatal infections (Tazi et al., 2010). The proteins responsible for the higher capacity of this ST-17 subset of strains to form biofilms have not yet been identified, and could be possible targets to prevent colonization/disease of this hypervirulent lineage.

Park et al. investigated the phenotype of the CsrRS twocomponent regulatory system knockout, and showed an increase capacity of CsrRS mutant bacteria to adhere to host cells and to form biofilm-like structures on plate (Park et al., 2012). This regulatory effect of CsrRS on bacterial adherence and biofilm formation correlated with the expression of multiple surface adhesins but not of Pilus 1, excluding a role of this pilus variant in biofilm formation in the investigated isolate. The same and other authors identified $\mathrm{BsaB} / \mathrm{FbsC}$ as a protein adhesin involved in biofilm formation and regulated by the CsrRS system (Buscetta et al., 2014; Jiang and Wessels, 2014); different from the pilus proteins, FbsC expression appeared slightly downregulated in a CsRS dependent manner at acidic versus neutral $\mathrm{pH}$.

\section{CONCLUSIONS}

Similar to other Gram-positive pathogens colonizing the human host, Group B Streptococcus can form multicellular communities that are expected to facilitate its persistence under environmental stress conditions. A number of in vitro studies have demonstrated that GBS forms biofilm-like structures on abiotic surfaces. Yet, the presence in these structures of the extracellular matrix typical of bacterial biofilms has not been investigated in this species. Additional studies are also needed to confirm the relevance of biofilm formation in vivo. If this would be the case, the involved virulence factors could constitute new therapeutic and preventive targets against this important human pathogen.

\section{REFERENCES}

Borges, S., Silva, J., and Teixeira, P. (2012). Survival and biofilm formation by Group B streptococci in simulated vaginal fluid at different pHs. Antonie Van Leeuwenhoek 101, 677-682. doi: 10.1007/s10482-011-9666-y
Buscetta, M., Papasergi, S., Firon, A., Pietrocola, G., Biondo, C., Mancuso, G., et al. (2014). FbsC, a novel fibrinogen-binding protein, promotes Streptococcus agalactiae-host cell interactions. J. Biol. Chem. 289, 21003-21015. doi: 10.1074/jbc.M114.553073

Carey, A. J., Tan, C. K., Mirza, S., Irving-Rodgers, H., Webb, R. I., Lam, A., et al. (2014). Infection and cellular defense dynamics in a novel 17beta-estradiol murine model of chronic human group B streptococcus genital tract colonization reveal a role for hemolysin in persistence and neutrophil accumulation. J. Immunol. 192, 1718-1731. doi: 10.4049/jimmunol.1202811

Dando, S. J., Mackay-Sim, A., Norton, R., Currie, B. J., St John, J. A., Ekberg, J. A., et al. (2014). Pathogens penetrating the central nervous system: infection pathways and the cellular and molecular mechanisms of invasion. Clin. Microbiol. Rev. 27, 691-726. doi: 10.1128/CMR.00118-13

Donlan, R. M., and Costerton, J. W. (2002). Biofilms: survival mechanisms of clinically relevant microorganisms. Clin. Microbiol. Rev. 15, 167-193. doi: 10.1128/CMR.15.2.167-193.2002

Dramsi, S., Caliot, E., Bonne, I., Guadagnini, S., Prevost, M. C., Kojadinovic, M., et al. (2006). Assembly and role of pili in group B streptococci. Mol. Microbiol. 60, 1401-1413. doi: 10.1111/j.1365-2958.2006.05190.x

D’Urzo, N., Martinelli, M., Pezzicoli, A., De Cesare, V., Pinto, V., Margarit, I., et al. (2014). Acidic pH strongly enhances in vitro biofilm formation by a subset of hypervirulent ST-17 Streptococcus agalactiae strains. Appl. Environ. Microbiol. 80, 2176-2185. doi: 10.1128/AEM.03627-13

Edwards, M. S., Rench, M. A., Palazzi, D. L., and Baker, C. J. (2005). Group B streptococcal colonization and serotype-specific immunity in healthy elderly persons. Clin. Infect. Dis. 40, 352-357. doi: 10.1086/426820

Froeliger, E. H., and Fives-Taylor, P. (2001). Streptococcus parasanguis fimbriaassociated adhesin fap1 is required for biofilm formation. Infect. Immun. 69, 2512-2519. doi: 10.1128/IAI.69.4.2512-2519.2001

Gibbs, R. S., Schrag, S., and Schuchat, A. (2004). Perinatal infections due to group B streptococci. Obstet. Gynecol. 104, 1062-1076. doi: 10.1097/01.AOG.0000144128.03913.c2

Hansen, S. M., Uldbjerg, N., Kilian, M., and Sorensen, U. B. (2004). Dynamics of Streptococcus agalactiae colonization in women during and after pregnancy and in their infants. J. Clin. Microbiol. 42, 83-89. doi: 10.1128/JCM.42.1.83-89.2004

Ho, Y. R., Li, C. M., Yu, C. H., Lin, Y. J., Wu, C. M., Harn, I. C., et al. (2013). The enhancement of biofilm formation in Group B streptococcal isolates at vaginal pH. Med. Microbiol. Immunol. 202, 105-115. doi: 10.1007/s00430-012-0255-0

Jiang, S., and Wessels, M. R. (2014). BsaB, a novel adherence factor of group B Streptococcus. Infect. Immun. 82, 1007-1016. doi: 10.1128/IAI.01014-13

Kaur, H., Kumar, P., Ray, P., Kaur, J., and Chakraborti, A. (2009). Biofilm formation in clinical isolates of group B streptococci from north India. Microb. Pathog. 46, 321-327. doi: 10.1016/j.micpath.2009.04.004

Keefe, G. P. (1997). Streptococcus agalactiae mastitis: a review. Can. Vet. J. 38, 429-437.

Konto-Ghiorghi, Y., Mairey, E., Mallet, A., Dumenil, G., Caliot, E., Trieu-Cuot, P., et al. (2009). Dual role for pilus in adherence to epithelial cells and biofilm formation in Streptococcus agalactiae. PLoS Pathog 5:e1000422. doi: 10.1371/journal.ppat.1000422 
Lauer, P., Rinaudo, C. D., Soriani, M., Margarit, I., Maione, D., Rosini, R., et al. (2005). Genome analysis reveals pili in Group B Streptococcus. Science 309, 105. doi: $10.1126 /$ science. 1111563

Madzivhandila, M., Adrian, P. V., Cutland, C. L., Kuwanda, L., Schrag, S. J., and Madhi, S. A. (2011). Serotype distribution and invasive potential of group B streptococcus isolates causing disease in infants and colonizing maternalnewborn dyads. PLoS ONE 6:e17861. doi: 10.1371/journal.pone.0017861

Manetti, A. G., Koller, T., Becherelli, M., Buccato, S., Kreikemeyer, B., Podbielski, A., et al. (2010). Environmental acidification drives S. pyogenes pilus expression and microcolony formation on epithelial cells in a FCT-dependent manner. PLoS ONE 5:e13864. doi: 10.1371/journal.pone.0013864

Manetti, A. G., Zingaretti, C., Falugi, F., Capo, S., Bombaci, M., Bagnoli, F., et al. (2007). Streptococcus pyogenes pili promote pharyngeal cell adhesion and biofilm formation. Mol. Microbiol. 64, 968-983. doi: 10.1111/j.13652958.2007.05704.x

Margarit, I., Rinaudo, C. D., Galeotti, C. L., Maione, D., Ghezzo, C., Buttazzoni, E., et al. (2009). Preventing bacterial infections with pilus-based vaccines: the group B streptococcus paradigm. J Infect. Dis. 199, 108-115. doi: 10.1086/595564

Martins, E. R., Andreu, A., Melo-Cristino, J., and Ramirez, M. (2013). Distribution of Pilus Islands in Streptococcus agalactiae that cause human infections: insights into evolution and implication for vaccine development. Clin. Vaccine Immunol. 20, 313-316. doi: 10.1128/CVI.00529-12

Moscoso, M., Garcia, E., and Lopez, R. (2006). Biofilm formation by Streptococcus pneumoniae: role of choline, extracellular DNA, and capsular polysaccharide in microbial accretion. J. Bacteriol. 188, 7785-7795. doi: 10.1128/JB.00673-06

O’Toole, G., Kaplan, H. B., and Kolter, R. (2000). Biofilm formation as microbial development. Annu. Rev. Microbiol. 54, 49-79. doi: 10.1146/annurev.micro. 54.1.49

Park, S. E., Jiang, S., and Wessels, M. R. (2012). CsrRS and environmental pH regulate group B streptococcus adherence to human epithelial cells and extracellular matrix. Infect. Immun. 80, 3975-3984. doi: 10.1128/IAI.00699-12

Rinaudo, C. D., Rosini, R., Galeotti, C. L., Berti, F., Necchi, F., Reguzzi, V., et al. (2010). Specific involvement of pilus type $2 \mathrm{a}$ in biofilm formation in group B Streptococcus. PLoS ONE 5:e9216. doi: 10.1371/journal.pone.0009216

Rosini, R., Rinaudo, C. D., Soriani, M., Lauer, P., Mora, M., Maione, D., et al. (2006). Identification of novel genomic islands coding for antigenic pilus-like structures in Streptococcus agalactiae. Mol. Microbiol. 61, 126-141. doi: 10.1111/j.1365-2958.2006.05225.x

Skoff, T. H., Farley, M. M., Petit, S., Craig, A. S., Schaffner, W., Gershman, K., et al. (2009). Increasing burden of invasive group B streptococcal disease in nonpregnant adults, 1990-2007. Clin. Infect. Dis. 49, 85-92. doi: 10.1086/ 599369

Soriani, M., and Telford, J. L. (2010). Relevance of pili in pathogenic streptococci pathogenesis and vaccine development. Future Microbiol. 5, 735-747. doi: $10.2217 /$ fmb. 10.37

Tazi, A., Disson, O., Bellais, S., Bouaboud, A., Dmytruk, N., Dramsi, S., et al. (2010). The surface protein HvgA mediates group B streptococcus hypervirulence and meningeal tropism in neonates. J. Exp. Med. 207, 2313-2322. doi: 10.1084/jem.20092594

Ton-That, H., and Schneewind, O. (2004). Assembly of pili in Gram-positive bacteria. Trends Microbiol. 12, 228-234. doi: 10.1016/j.tim.2004.03.004

Yang, Q., Porter, A. J., Zhang, M., Harrington, D. J., Black, G. W., and Sutcliffe, I. C. (2012). The impact of $\mathrm{pH}$ and nutrient stress on the growth and survival of Streptococcus agalactiae. Antonie Van Leeuwenhoek 102, 277-287. doi: 10.1007/s10482-012-9736-9

Conflict of Interest Statement: Dr. Roberto Rosini and Dr. Immaculada Margarit are Novartis Vaccine employees. Dr. Immaculada Margarit holds Novartis stock options.

Received: 10 December 2014; accepted: 14 January 2015; published online: 04 February 2015.

Citation: Rosini R and Margarit I (2015) Biofilm formation by Streptococcus agalactiae: influence of environmental conditions and implicated virulence factors. Front. Cell. Infect. Microbiol. 5:6. doi: 10.3389/fcimb.2015.00006

This article was submitted to the journal Frontiers in Cellular and Infection Microbiology.

Copyright (C) 2015 Rosini and Margarit. This is an open-access article distributed under the terms of the Creative Commons Attribution License (CC BY). The use, distribution or reproduction in other forums is permitted, provided the original author(s) or licensor are credited and that the original publication in this journal is cited, in accordance with accepted academic practice. No use, distribution or reproduction is permitted which does not comply with these terms. 